\title{
Editorial
}

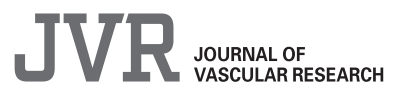

\section{We Can Do It!}

I want to begin by noting how proud and grateful we are to Prof. Ulrich Pohl for the hard work and loyalty he has provided for the many years as Editor in Chief of the Journal of Vascular Research (JVR). We can only hope that in the succeeding years we will be as influential and successful as he has been. Thank you Ulli!

Moving forward, there are many things to be proud of at JVR, including its long history in the field ( $>50$ years), the publication by JVR of many landmark papers in vascular biology, its close association with the European Society for Microcirculation (ESM), and the fact that it is completely run by and for vascular biology researchers and clinicians. We can look at each of these components as important reasons to believe in the viability, outreach, and quality of JVR.

However, the journal is facing major pressure, especially in terms of the impact factor, the (unfortunate) yardstick by which journals are measured for promotion, tenure, and perceived impact. This is not a problem JVR can hide from, especially if we wish to maintain and expand the journal to authors and maintain the high quality of JVR publications. For this reason, we have begun to organize an aggressive plan to address this issue. It may take a couple of years to bear fruit, but I believe it is a plan we can all get behind and help with.

Two "R's" will be especially important to begin with: Rigor and time to Review. To begin with Rigor, we want the readers of JVR to be confident in the data they are presented. Starting over the next few months, we will have enhanced plagiarism checks of manuscripts, and we will begin requiring data presented for each individual experiment in bar graphs, as well as uncropped western blot presentation (to name just a few new data presentation items). We don't do this in response to any particular incident, but to ensure as much trust as possible between the JVR reader and the author.

As scientists, we are all keenly aware of the time it takes to move a paper through the peer-review process. For this reason, we are focusing on greatly expediating the time to first review, revision, and publication. We have already begun acceleration by processing a triaged manuscript within $36 \mathrm{~h}$, and our goal is to have the time to first review in under 12 days by the end of 2020 . We are excited what progress we are making in this area. On the reviewer end, thoughtful and timely critiques are essential to our journal quality, but we realize this takes time away from science. For this reason, we will begin to incentivize reviewers for timely and thoughtful reviews. More information on this will be forthcoming.

These are the first plans as I move into JVR, with more to come, including the strong relationship with ESM, and more reviewer transparency. I am excited about the future of JVR, and I believe we can do it! We will move JVR forward in a meaningful way, and I would like everyone to be a part of it.

Brant E. Isakson, Charlottesville 